\title{
Dietary Interventions: A Promising Treatment for Polycystic Ovary Syndrome
}

\author{
Xuan Che ${ }^{a, b}$ Zhuo Chen ${ }^{c}$ Mingqi Liu $^{b}$ Zhongcheng Mob,c \\ ${ }^{a}$ Clinical Anatomy \& Reproductive Medicine Application Institute, Hengyang Medical School, University of \\ South China, Hengyang, China; ${ }^{b}$ Guangxi Key Laboratory of Diabetic Systems Medicine, Guilin Medical University, \\ Guilin, China; ' Guangxi Province Postgraduate Cotraining Base for Cooperative Innovation in Basic Medicine \\ (Guilin Medical University and Yueyang Women \& Children's Medical Center), Yueyang, China
}

\section{Keywords}

Polycystic ovary syndrome · Dietary interventions · Dietary pattern

\begin{abstract}
Background: Dietary interventions as a first-line treatment for patients with polycystic ovary syndrome (PCOS) have been evaluated, but the optimal diet has not been determined. Proper diet and the maintenance of adequate nutritional status are of great importance in the prevention of this disorder, and therapeutics and dietary habits play an important role in the recovery of patients with PCOS. Summary: A range of dietary patterns have been shown to impact weight loss and insulin resistance (IR) and improve reproductive function, including the Mediterranean diet, the ketogenic diet, Dietary Approaches to Stop Hypertension, and other dietary patterns. Key Messages: Diets that can reduce rates of obesity and IR are beneficial to women with PCOS, the status of obesity and IR should be determined at the early stage of the disease, so as to develop individualized and sustainable dietary intervention. The long-term efficacy, safety, and health benefits of diet management in patients with PCOS need to be tested by further researches.
\end{abstract}

\section{Introduction}

Polycystic ovary syndrome (PCOS) is a complicated and common endocrine-metabolic disorder of unknown etiology that affects, according to different diagnostic criteria, $5-20 \%$ of women of reproductive age worldwide [1, $2]$. Women with PCOS often present with clinical or biochemical hyperandrogenism, oligoanovulation, and/or polycystic ovarian morphology, and these patients may present to clinicians with a variety of manifestations, including infertility, hirsutism, acne, insulin resistance (IR), and dyslipidemia, among others [3,4]. As a result of the life-long pathological state of PCOS, patients with PCOS are at increased risk of obesity, metabolic dysfunction, vascular dysfunction, malignancy, reproductive complications, and mood disorders $[5,6]$.

Internationally, most studies indicate that women with and without PCOS have different baseline dietary intakes; the former with a higher intake of calories and saturated fat and inadequate fiber consumption. Clinical symptoms and the compounded risk of chronic disease in patients with PCOS may be exacerbated by these altera-

Xuan Che and Zhuo Chen contributed equally to this work. karger@karger.com

www.karger.com/anm (c) 2021 S. Karger AG, Basel
Correspondence to:

Zhongcheng Mo, zhchmo@ hotmail.com

\section{Karger"}


tions in diet. In addition, Szczuko et al. [7] analyzed the diet of 54 women of childbearing age with PCOS and demonstrated that metabolic disorders related to improper ovarian function are due to unhealthy diets in women with PCOS. It is important to note that Tremellen and Pearce [8] proposed a microbiological hypothesis for how PCOS occurs, suggesting that a poor diet leads to disorders of the intestinal flora, and the dysbiosis of gut microbiota theory can explain all 3 components of the syndrome. In line with those studies, traditional Chinese medicine also argues that PCOS is associated with dietetic abnormalities: a poor diet high in fat, sugar, and grease can damage the spleen and stomach, leading to spleen wet-sheng and phlegm within the knot, followed by blood stagnation and dampness (accumulation in the ovaries and uterus), which can be the result of oligo-ovulation [9, 10]. Epidemiological studies and large clinical trials have identified long-lasting, low-grade chronic inflammation in PCOS and potential anti-inflammatory and pro-inflammatory components in diets associated with the pathogenesis of PCOS. An unhealthy diet is a key component of the "deadly quartet" of metabolic risk factors associated with PCOS pathophysiology, along with lowgrade inflammation, hyperinsulinemia, and hyperandrogenism [11]. The prevalence of eating disorders is increased in PCOS and suggests that all women with PCOS should be routinely screened at the time of diagnosis [12]. According to the newly published international evidence-based guidelines for the assessment and management of PCOS, general healthy eating principles should be followed for all women with PCOS throughout their lives, but for women with PCOS who are also overweight or obese, a variety of balanced dietary approaches can be recommended to reduce dietary energy intake and induce weight loss [13].

Researchers are committed to finding a diet that may aid in the prevention and treatment of PCOS. This review aimed to summarize the potential mechanisms, advantages, limitations, and currently available evidence for dietary interventions as a treatment for PCOS.

\section{Dietary Patterns and PCOS}

\section{Mediterranean Diet}

The Mediterranean diet (MedDiet) is recognized as the healthiest dietary model and has been included in international guidelines among recommended healthy dietary patterns because of its unique characteristics, including regular consumption of unsaturated fat, fiber, low-glycemic index (low-GI) carbohydrates, antioxidants, and vitamins, as well as appropriate amounts of animal-derived protein [14]. Numerous studies over several decades have shown that the adoption of the MedDiet pattern can protect against IR-related diseases, such as obesity, cardiovascular disease, type 2 diabetes (T2D), nonalcoholic fatty liver disease (NAFLD), cognitive impairment, breast cancer, and chronic kidney disease. The beneficial mechanisms of the MedDiet involve reducing inflammatory and oxidative stress markers and improving lipid profiles, insulin sensitivity, and endothelial function, as well as antiatherosclerotic and antithrombotic properties $[15,16]$. In addition, the MedDiet pattern is also regarded as the primary prevention of metabolic syndrome (MetS) [17]. In consideration of the tight relationship between PCOS and obesity, low-grade chronic inflammation and IR, it appears likely that the MedDiet is one of the optimal nonpharmacological strategies for the treatment of PCOS.

One recent study investigated the association between PCOS clinical severity, body composition, and adherence to the MedDiet and showed that women with PCOS had low consumption of extra-virgin olive oil, legumes, fish, and nuts compared to controls; consumed more simple carbohydrates, saturated fatty acids, total fat, polyunsaturated fatty acids (PUFAs), and n-6 PUFAs; and consumed fewer complex carbohydrates, fiber, n-3 PUFAs, and monounsaturated fatty acids than a control group. Meanwhile, testosterone levels showed significant correlations with the dietary nutrients mentioned above: there were positive associations with C-reactive protein levels, simple carbohydrates, saturated fatty acids, PUFAs, and n-6 PUFAs, and negative correlations with consumption of protein, complex carbohydrates, fiber, n-3 PUFAs and monounsaturated fatty acids. Among them, n-3 PUFAs supplementation has been proven to improve insulin sensitivity and is beneficial to metabolic management [18]. A negative correlation was found between the degree of adherence to the MedDiet and the clinical severity of PCOS; furthermore, an unhealthy diet in women with PCOS contributed to more severe inflammation, IR, and hyperandrogenemia [14]. Another recent study found that this imbalance in patients consuming a predominantly Western diet with high intake of n-6 PUFAs, sugars, and meat and low consumption of fruits and vegetables led to an impaired gut epithelial barrier and disturbances in the intestinal microbiota, which can bring on chronic mucosal inflammation, while the MedDiet has been proven to improve inflammation by acting on intestinal permeability, gut microbiota composition, and in- 


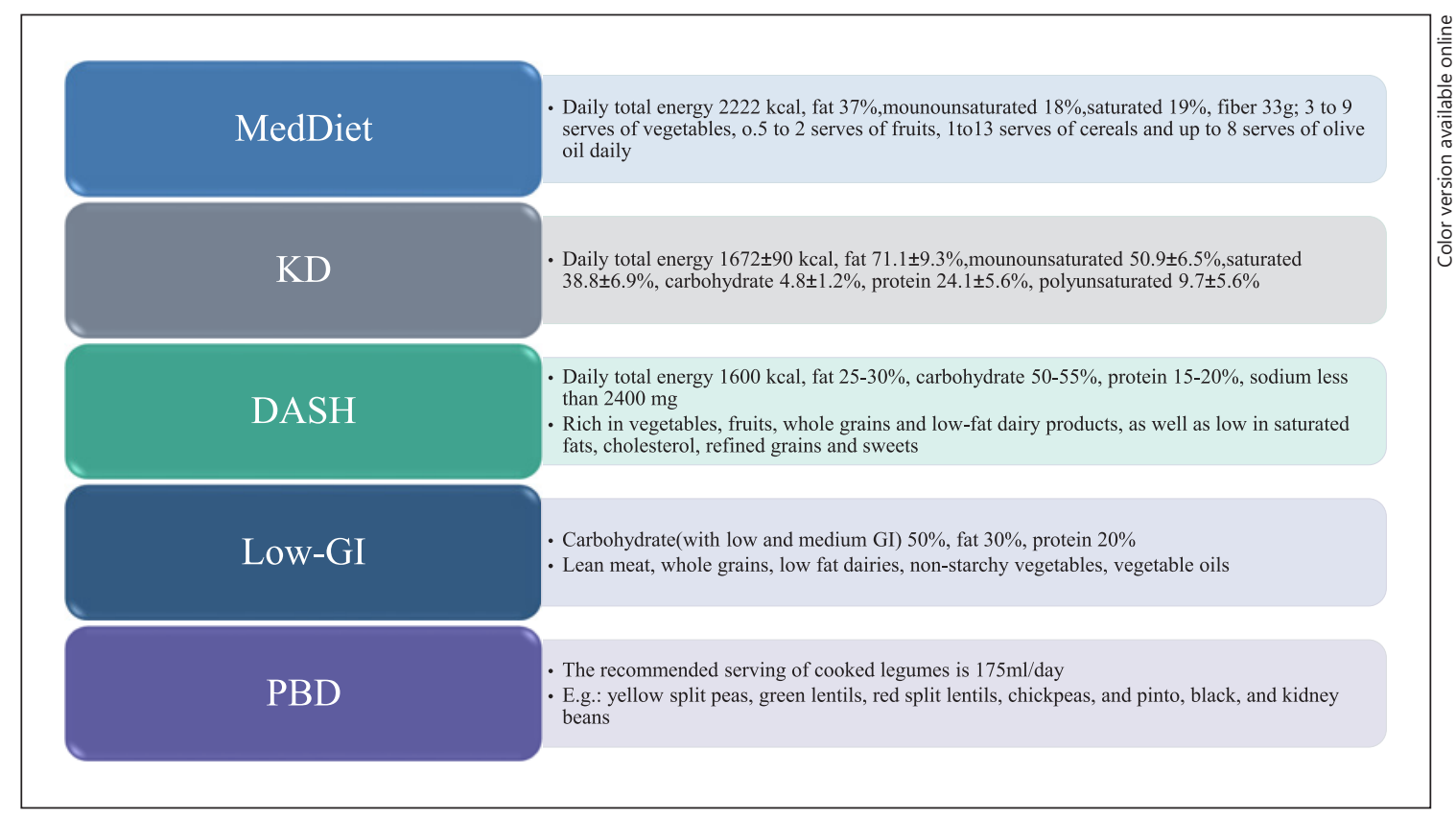

Fig. 1. General content and characteristic of dietary patterns. MedDiet, Mediterranean diet; KD, ketogenic diet; DASH, dietary approaches to stop hypertension; low-GI, low-glycemic index diets; PBD, pulse-based diet.

flammatory responses [19]. Moreover, the latest research shows that adherence to the MedDiet can improve ovarian form (ovarian volume and follicle number per ovary) by impacting obesity, IR, and hyperandrogenism [20].

The beneficial effects of the MedDiet have been attributed to plant polyphenols. Plant polyphenols obtained from vegetables, fruits, legumes, grains, nuts, seeds, and particularly red wine and extra-virgin olive oil in the MedDiet can be useful to combat MetS and have been scientifically investigated over the last several decades [21]. Extra-virgin olive oil is regarded as the main source of fat and predominant health-promoting component of the MedDiet. Polyphenols are present abundantly in extra-virgin olive oil, and many available data and population studies suggest that the presence of substantial amounts of polyphenols are key health-promoting components of the MedDiet. Polyphenols have a possible role in disease prevention and therapeutic potential in women with PCOS by slowing the progression of inflammation and improving both insulin sensitivity and compensatory hyperinsulinemia. Vitamin E and oleic acid are also primary functional components of olive oil, and their gene regulation properties have shown their predicted therapeutic applications in chronic inflammation and cancer [22]. Furthermore, they may help reduce the risk of PCOS-related cancers. In addition, MedDiets are particu- larly rich in resveratrol, a natural polyphenol found in grapes, berries, and wine, which reduces androgen production and ameliorates hyperandrogenism in PCOS [17].

While medical professionals appear to recognize the health benefits of the MedDiet, it is still an enormous challenge to implement this protective dietary pattern in areas outside of the Mediterranean region [23]. Davis et al. [24] formed a comprehensive and quantitative definition of the MedDiet by summarizing existing definitions and calculating the mean food groups and nutrients (Fig. 1). This definition is intuitively clear and easy for most people to comprehend, and it might improve adherence to the MedDiet in women with PCOS. Furthermore, based on the latest scientific evidence on nutrition and health, the "Mediterranean Diet Foundation Expert Group" has developed a MedDiet pyramid, which is not only a dietary model but also represents cultural heritage of a lifestyle that has been passed over many centuries, emphasizing a healthy lifestyle that includes adequate rest, culinary activities, conviviality, moderation, frugality, and physical exercise [25]. This new graphic representation might help improve adherence to the MedDiet and its way of life, and it should be recommended without any restrictions to promote health and well-being in women with PCOS. 


\section{Ketogenic Diet}

The ketogenic diet (KD), or keto diet, is a high-fat and low-carbohydrate diet that encourages forgoing nearly all carbohydrates, consuming high levels of fat (generally exceeding $70 \%$ of calories consumed), and avoiding excess protein, resulting in high production of ketones (principally acetoacetate and $\beta$-hydroxybutyrate) and nutritional ketosis. The KD is known for its antiepileptic effects in the treatment of refractory epilepsy [26]. KD has also been proposed as an effective treatment for other neurological disorders, including Alzheimer's disease, Parkinson's disease, and autism.

Ketone metabolism is a central node that could be affected by physiological homeostasis [27]. Therefore, researchers have noted that KD has treatment implications not only for diseases of the nervous system but also for diseases of the gastrointestinal and endocrine systems, particularly T2D and obesity but also NAFLD and nonalcoholic steatohepatitis [28]. A study in humans from Canada confirmed that plasma ketones increased 8-fold while plasma glucose decreased by $24 \%$ in healthy adults during a very high-fat equicaloric KD [29]. KD not only decreases postprandial insulin secretion but also reverses IR by inducing weight loss and loss of fat mass, thereby helping restore the functionality of insulin [30]. The metabolic and endocrine effects of $\mathrm{KD}$ are mainly embodied in the substantial reduction in weight, fasting serum insulin, percent of free testosterone, and the luteinizing hormone ( $\mathrm{LH}) /$ follicle-stimulating hormone ratio in women with obesity and PCOS [31]. A pilot study has shown that adherence to a low-carbohydrate KD for 6 months could decrease androgen secretion, increase sex hormone-binding globulin (SHBG), and improve insulin sensitivity, thereby renormalizing endocrine function, which has beneficial effects in the treatment of women with PCOS [32]. Paoli et al. [33] studied 14 overweight women with a diagnosis of PCOS who observed a modified KD (Fig. 1). Twelve weeks after dietary intervention, those women had a significant reduction in body weight, body mass index (BMI), fat mass, and visceral adipose tissue, along with a marked improvement in IR. Moreover, almost all the parameters related to PCOS improved, including the LH/follicle-stimulating hormone ratio and levels of total LH, free testosterone, SHBG, dehydroepiandrosterone sulfate, estradiol, and progesterone. These results seem to suggest that $\mathrm{KD}$ is the optimal dietary intervention for patients with PCOS.

Although a short-term KD has achieved the desired effect, PCOS is a chronic disease requiring long-term treatment, and animal experiments suggest that long- term maintenance of a KD affects metabolic state and stimulates the development of NAFLD and systemic glucose intolerance [34]. Another study demonstrated that KD contributed to severe hepatic lipid accumulation, steatosis, and fibrosis by driving alterations in inflammation, lipid metabolism, and hepatic glucose levels in a mouse model of diabetes; therefore, there is also deep concern over the potential side effects of the high-fat intake of $\mathrm{KD}$, which should be considered carefully in the long-term application of KD [35]. Considering the potential side effects of a high-fat diet, a very-low-calorie KD (VLCKD) has attracted wide attention from researchers. A low lipid count is a characteristic of VLCKD, and the lipids are mainly derived from olive oil $(\simeq 20$ g per day) [36], which is quite similar to the MedDiet. In 2016, the Italian Association of Dietetics and Clinical Nutrition and the Italian Society of Obesity proposed VLCKD as a therapeutic option for drug-resistant epilepsy, NAFLD, and obesity associated with comorbidities, as well as a useful tool for weight loss before bariatric surgery. Obesity is common in patients with PCOS and can further aggravate their symptoms; therefore, VLCKD may help these patients lose weight and improve their symptoms. A randomized pilot study comparing VLCKD based on whey, vegetable protein, and animal proteins in patients with obesity and IR indicated that 45 day-long VLCKD improved lipid metabolism, glycemic control, and arterial pressure, and caused a profound reduction in body weight; additionally, whey and vegetable proteins have a safer profile and resulted in healthier gut microbiota [37]. This improvement is very beneficial for patients who need long-term dietary intervention. While the modified $\mathrm{KD}$ may be a valuable treatment for PCOS, more tests are needed to verify its long-term effects.

It has been reported that the levels of carbohydrate tolerance vary greatly from person to person and even within the same individual; therefore, ketosis (blood ketone bodies $>0.5 \mathrm{mM}$ ) may not occur even in VLCKD [38]. In this regard, proper clinical investigations must be conducted, and a systematic evaluation of KD or VLCKD treatment for PCOS is urgently needed.

\section{Dietary Approaches to Stop Hypertension}

The Dietary Approaches to Stop Hypertension (DASH) dietary pattern, a low-GI and low energy-dense diet, was primarily designed for lowering blood pressure and emphasizes fruits, vegetables, whole grains, nuts, legumes, and fat-free/low-fat dairy products while recommending low consumption of saturated fats, cholesterol, red and processed meats, refined grains, and sweets. This 
diet results in the consumption of higher amounts of dietary fiber, folic acid, phytoestrogens, potassium, magnesium, and other beneficial nutrients [39, 40]. Specifically, the DASH diet includes 4-5 servings of vegetables and fruit per day, 3 servings of low-fat dairy per day, 6-8 servings of whole grains per day, and less than 6 servings of meat, poultry, and fish per day (Fig. 1). It also advises 4-5 servings per week of nuts, seeds, and beans, and encourages the consumption of foods with high potassium and fiber, low saturated fat, and low sodium [41]. Although DASH was first suggested as a diet that is beneficial for people with hypertension, its beneficial effects have also been reported in IR [39], increased inflammation [42], obesity [43], T2D, gestational diabetes mellitus, MetS, NAFLD, and cardiovascular disease [44]. International diabetes and heart association guidelines also highly recommend the DASH dietary pattern [41].

The DASH diet contains a higher number of antioxidants, magnesium, and fiber than other low-GI diets, which might further influence the abnormal metabolic profile and IR in women with PCOS. Furthermore, its high content of calcium, folate, and other beneficial nutrients might have potential favorable effects on IR and inflammation in PCOS. A randomized controlled trial found that consumption of a DASH diet for 8 weeks in overweight or obese patients with PCOS had an effect on lipid profiles and biomarkers of oxidative stress, including plasma total antioxidant capacity and total glutathione; furthermore, it also led to the improvement of abdominal fat accumulation, IR, and serum hs-C-reactive protein levels $[39,45]$. In another study by Foroozanfard et al. [46]., it was observed that adherence to the DASH diet for 12 weeks among women with PCOS may confer advantageous therapeutic potential by reducing BMI and exerting beneficial effects on serum anti-Mullerian concentrations (a marker of ovarian dysfunction), markers of insulin metabolism, SHBG, plasma nitric oxide, free androgen index, and malondialdehyde levels. Another 3-month study by Azadi-Yazdi et al. [47] found that the DASH diet in overweight women with PCOS resulted in improved weight loss, BMI, and fat mass; furthermore, it resulted in a significant increase in SHBG and antioxidant status and a marked decrease in levels of androstenedione, independent of its weight loss effects. It has been reported that the DASH diet might be the optimal choice to improve IR and weight loss in women with PCOS [48].

\section{Low-GI Diets}

Low-GI diets are defined as those that obtain most carbohydrates from low-GI sources (Fig. 1). Foods having carbohydrates that are digested, absorbed, and metabolized slowly are regarded as low-GI foods [49]. Higher postprandial glycemia increases the risk of chronic lifestyle-related diseases, which is a universal mechanism for disease progression, and carbohydrates are the main dietary component affecting insulin secretion and postprandial glycemia. Therefore, glycemic index (GI) is widely recognized to have clinical and public health significance because higher blood glucose concentrations and a greater demand for insulin have been produced by the same amount of carbohydrate; nevertheless, high glycemic index (high-GI) diets may directly increase IR through their effect on glycemia, free fatty acids, and counterregulatory hormone secretion [50], which suggests that the GI of contributing carbohydrate foods is important irrespective of the level of carbohydrate intake. Accumulating evidence indicates that low-GI diets aid in glycemic control [51], lipid profile control, and weight loss. They are also recommended in the primary prevention of MetS [52] and can reduce the risk of chronic diseases, such as T2D and coronary heart disease [53]. As suggested above, the use of these markers of carbohydrate food is very valuable in dietary guidelines for the general population.

Low-GI diets are becoming more acceptable to both registered dietitians and patients in the management of PCOS. Dietary GI is enhanced in patients with the classic PCOS phenotype and is related to a worse anthropometric and metabolic profile. Adiposity and the classic PCOS phenotype are predictors of higher dietary GI unrelated to age [54]. Compared with healthy women, a lower concentration of glutathione peroxidase was observed in PCOS patients, and Szczuko et al. [55] suggested that low-GI dietetic intervention decreases inflammation in women with PCOS by increasing the concentration of uric acid and the activity of glutathione peroxidase. Moreover, a study from Australia compared changes in insulin sensitivity as a clinical outcome after comparable weight loss with consumption of low-GI diets compared with a conventional healthy diet in women with PCOS, providing objective evidence to justify the use of low-GI diets in the management of PCOS [56]. Short-term low-GI dietary modification could slightly increase insulin sensitivity in women with PCOS [57]. In addition, compared with a normal GI diet, a low-GI diet improves ovulation cycles in patients with PCOS and anovulation [58]. A preliminary study reported that a low-GI diet might reduce endometrial cancer risk by decreasing endometrial thickness and increasing the number of menstrual cycles in women with PCOS [59]. In recent years, researchers have attached a 
great deal of value to the effects of low-GI diets on weight loss and on the metabolic changes that result from obesity. In women with overweight and obesity, a low-GI diet combined with a hypocaloric diet seems to be more beneficial; hypocaloric low-GI diets reduced BMI, percentage of body fat, and leptin concentrations, and improved oocyte development and fertility rates [60]. A recent study viewed low-GI diets as an optimal dietary choice for women with PCOS owing to their high rates of adherence and efficacy in improving common clinical manifestations of PCOS, including IR, hyperandrogenism, hirsutism, acne, and menstrual irregularities. In addition, 1 study showed that a low-GI diet has equally beneficial effects on anthropometric and metabolic characteristics of overweight women with and without PCOS [61].

An earlier cohort study suggests similarities in the consumption of total energy, high-GI foods, micronutrients, and macronutrients between women with PCOS and healthy controls, but the former consumes a large number of specific foods with a high GI, such as white bread and fried potatoes [62]. Furthermore, a case-control study comparing the dietary habits of women with PCOS with age-BMI matched controls also showed no difference in energy or macronutrient intake among women with PCOS and healthy women; however, their results indicated a higher intake of high-GI food items and a lower consumption of vegetables and legumes in women with PCOS [63]. Considering the results of these studies, it seems reasonable to conclude that women with PCOS had a dietary pattern that was characterized by higher consumption of high-GI food items in general, which could further support the therapeutic and signatory role of low-GI diets in PCOS. Continued identification of low-GI foods will make the implementation of low-GI diets easier and more universal. However, the practical applicability of the value of GI remains somewhat contentious. Matthan et al. [64] suggested that GI could not be a reliable approach to guiding food choices because of the high degree of variability in individuals.

\section{Pulse-Based Diet}

Pulses (e.g., lentils, chickpeas, split peas, and drybeans) are high in fiber and low in fat, contain high-quality protein and complex carbohydrates with a low GI and are a significant source of vitamins and minerals, such as iron, zinc, folate, calcium, magnesium, and potassium [65]. Pulses, alone or as part of low-GI or high-fiber diets, improve markers of longer term glycemic control in humans [66], and the pulse diet (Fig. 1) has been used in clinical populations to improve or maintain insulin sensitivity and prevent or reduce IR [67]. Pulse consumption has been shown to improve cardiometabolic disease risk factors in women with PCOS [68]. However, it is even more noteworthy that the phytochemicals, saponins, and tannins found in pulses have significant anticancer effects; in addition, adequate folate intake could reduce the risk of endometrial carcinoma, and the risk of endometrial carcinoma in women with PCOS is increased from 2 to 6-fold [69]. Thus, the possible positive effects of a pulsebased diet in women with PCOS are worth further study.

\section{Conclusions and Perspectives}

\section{Comparison of Dietary Interventions}

Several dietary patterns have been proposed for women with PCOS, and the characteristics and average nutrient content of these diets are shown in Figure 1, Table 1 have summarized the key health-promoting components of the various dietary patterns. Dietary interventions as a treatment for PCOS may improve reproductive and metabolic function, and the proposed mechanisms of those dietary patterns in PCOS are compared in Figure 2.

Diets that can improve obesity and IR are beneficial to patients. Weight loss improved the presentation of PCOS regardless of dietary composition in the majority of dietary studies. Adiponectin is the most abundant adipokine secreted by adipocytes and involved in the production of steroid hormones, glucose homeostasis, and other functions [70]. Women with PCOS, when gaining weight that results in obesity, can aggravate the phenotype of menstrual abnormalities, infertility, anovulation, and metabolic alterations [71]. Women with PCOS have an equal resting metabolic rate to that of women without PCOS, indicating that the ability to lose weight in terms of energy metabolism does not differ between women with and without PCOS; however, patients seen in the clinical setting are often more severely hyperandrogenic, have higher rates of obesity, and are more anxious about weight and dieting than women with PCOS detected in epidemiological studies [72]. Adipose tissue is an extraglandular source of androgens, and obesity, especially abdominal obesity, can worsen hyperandrogenism [33]. A recent study demonstrated that increased BMI is causal for PCOS, and obesity influences the development of PCOS [73]. Diet-induced weight loss of at least 5\% contributes to reducing levels of insulin and androgens, restoring ovulation and increasing fertility rates [36]. IR is a very common feature in women with PCOS, especially in patients with obesity [74]. Previous animal and human 
Table 1. Key health-promoting components of the various dietary interventions

\begin{tabular}{lll}
\hline Dietary & Key components & Function \\
\hline MedDiet & Extra-virgin olive oil & The main source of health-promoting component \\
\cline { 2 - 3 } & Polyphenols & $\begin{array}{l}\text { Slow the progression of the inflammatory status, and improve } \\
\text { both insulin sensitivity and compensatory hyperinsulinemia }\end{array}$ \\
\cline { 2 - 3 } & Vitamin E and oleic acid & Treat chronic inflammation and cancer \\
\cline { 2 - 3 } & Resveratrol & Reduce androgen production \\
\hline KD & Nutritional ketosis & Decrease androgen secretion, increase SHBG, improve insulin \\
& Whey or vegetable protein & sensitivity, and renormalize endocrine functions \\
\hline DASH & Antioxidants, folic acid, magnesium, and dietary fiber & Healthier gut microbiota \\
\cline { 2 - 3 } & Calcium and folate & Improve the abnormal metabolic profile and IR. \\
\hline Low-Gl & Low-Gl foods (Gl $\leq 55$ on the glucose scale) & Improve common clinical manifestations of PCOS \\
\hline PBD & Phytochemicals, saponins, and tannins & Significant anticancer effects \\
\cline { 2 - 3 } & Adequate folate & Reduce the risk of endometrial carcinoma
\end{tabular}

MedDiet, Mediterranean diet; KD, ketogenic diet; DASH, dietary approaches to stop hypertension; low-GI, low-glycemic index; PBD, pulse-based diet; IR, insulin resistance; SHBG, sex hormone-binding globulin; PCOS, polycystic ovary syndrome.

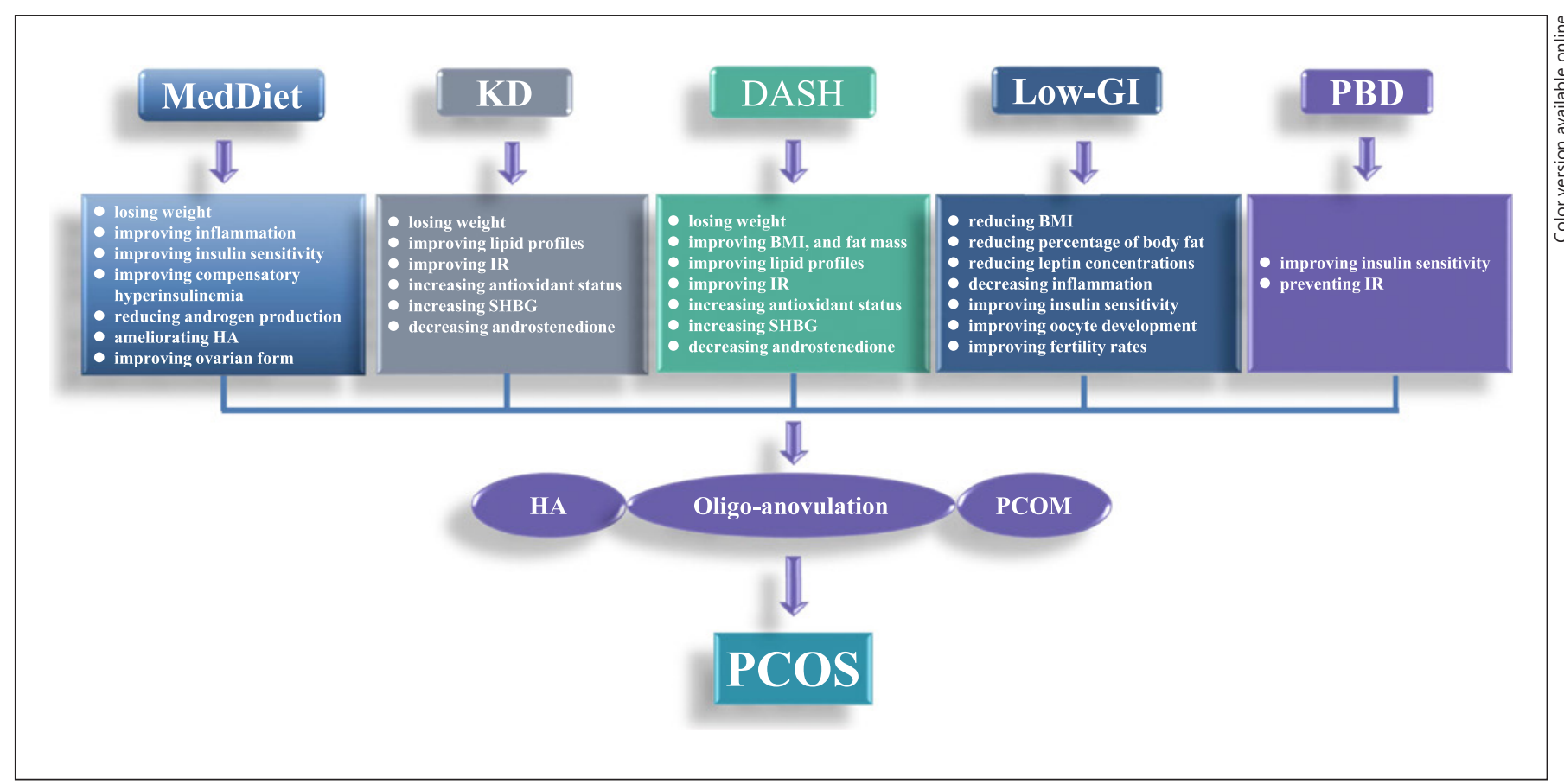

Fig. 2. The beneficial mechanisms of dietary patterns in women with PCOS. MedDiet, Mediterranean diet; KD, ketogenic diet; DASH, dietary approaches to stop hypertension; low-GI, low-glycemic index; PBD, pulse-based diet; HA, hyperandrogenism; IR, insulin resistance; SHBG, sex hormone-binding globulin; BMI, body mass index; PCOM, polycystic ovarian morphology; PCOS, polycystic ovary syndrome.

Dietary Interventions for Polycystic Ovary Syndrome 
studies revealed that IR and compensatory hyperinsulinemia lead to androgen excess in PCOS [75]. Androgen exposure and IR interact as both cause and effect under the action of the AKR1C3 enzyme in subcutaneous adipocytes, resulting in a vicious cycle [76]. Increased understanding of the role of IR in the pathogenesis of PCOS has led to the development of new strategies. A meta-analysis [77] reviewed 30 trials and revealed that diets low in carbohydrates $(<50 \%)$ are associated with better IR reduction in women with severe IR; therefore, it is postulated that low-carbohydrate diets can improve IR in patients with PCOS. However, this effect was more prominent for subjects with severe IR; thus, it is important to determine IR status early in the disease process. Overall, we determined that weight management and caloric restriction can improve IR in PCOS patients.

If we combine all the advantages of dietary changes, can we propose a new diet that can better guide patients with PCOS? Based on the MedDiet, appropriately reducing carbohydrate intake, choosing carbohydrates that are as low GI as possible, and increasing the intake of fresh vegetables, fruits, and nuts may help guide the diet of patients with PCOS. Further research is needed to determine a more accurate diet ratio and provide individualized dietary guidance for women with PCOS.

\section{Sustainability of Dietary Interventions}

The beneficial effect of dietary interventions on PCOS remains to be studied, and some researchers have begun to consider the sustainability of dietary interventions. Considering the side effects of medication, some patients may prefer to choose dietary interventions that have never shown side effects comparable to those seen in many drugs, but these changes are not sustainable for many patients. The long-term outcomes of the effect of dietary interventions on major clinical endpoints will be of importance [78] because only sustained, long-term dietary patterns can modify chronic disease risk, and long-term adherence to healthy dietary patterns is difficult to achieve. A randomized controlled trial [68] found a high rate $(33.7 \%)$ of participant attrition; similarly, the same high attrition rates (27-49\%) are found in other studies focused on lifestyle changes in women with PCOS, with a progressive increase in withdrawal corresponding to the duration of the intervention. Their observations highlight the importance of a multidisciplinary team and regular followup visits. However, a study from Australia [79] reported that women with PCOS had a healthier diet than controls, with lower saturated fat and GI intake and higher micronutrient intake, despite higher energy intake overall.
Additionally, a community cohort study [80] from Australia analyzed data from $n=7,569$ women $(n=414$ were women with PCOS and $n=7,155$ were women without PCOS) and found that women with PCOS were apt to consume a healthier dietary pattern. This positive finding may indicate that the diagnosis of PCOS contributes to an improvement in the quality of dietary intake, which might reflect the benefit of receiving clinical services and an awareness of the importance of eating a healthy diet. Additionally, strategies to promote reduced feelings of hunger, cravings, and deprivation may increase adherence to dietary prescriptions [81]. For instance, one of the clinical benefits of KD is appetite suppression [82], and VLCKD are increasingly established as a successful nutritional pattern to manage obesity; this is due to rapid weight loss that gives rise to positive psychological feedback, which in turn increases compliance with the diet [83]. Long-term adherence to dietary prescriptions is critical for weight management, preventing or delaying the occurrence of comorbidities, and improving the overall prognosis associated with PCOS; therefore, there is an urgent need for more patient-centered trials measuring the compliance of dietary interventions in women with PCOS.

In conclusion, all women with PCOS should understand that healthy lifestyle behaviors, healthy eating, and regular physical activity can improve health and well-being throughout life by optimizing hormonal outcomes, general health, and quality of life. However, there is still a lack of sufficient data to support the long-term efficacy, safety, and health benefits of these diets, and it is necessary to test and verify the effects of long-term dietary management of PCOS through further studies. Last, the dietary management of women with PCOS should be under the common guidance of registered dietitians, gynecologists, fertility specialists, and endocrinologists from the time of diagnosis to help patients recognize the role of diet and lifestyle factors in the disorder and how they may be changed through nonpharmacological means to improve prognosis.

\section{Acknowledgment}

The authors are grateful to Hongliang Zhu for language revision.

\section{Conflict of Interest Statement}

The authors declare no conflicts of interest. 


\section{Funding Sources}

This study was supported by Guangxi Natural Science Foundation under Grant No. 2020GXNSFAA238008, the Horizontal Cooperation project with Yueyang Women \& Children's Medical Center, and the Foundation for Guangxi Key Laboratory of Diabetic Systems Medicine (20-065-77).

\section{Author Contributions}

X.C. and Z.C. wrote the article. M.L. contributed to revision of the manuscript. Z.M. contributed to the design and revision of the manuscript. All authors were involved in editing the manuscript.

\section{References}

1 Azziz R, Carmina E, Chen Z, Dunaif A, Laven JS, Legro RS, et al. Polycystic ovary syndrome. Nat Rev Dis Primers. 2016 Aug 11;2:16057.

2 Azziz R. Polycystic ovary syndrome. Obstet Gynecol. 2018 Aug;132(2):321-36.

3 Yildiz BO, Bolour S, Woods K, Moore A, Azziz R. Visually scoring hirsutism. Hum Reprod Update. 2010 Jan-Feb;16(1):51-64

4 Yau TT, Ng NY, Cheung LP, Ma RC. Polycystic ovary syndrome: a common reproductive syndrome with long-term metabolic consequences. Hong Kong Med J. 2017 Dec;23(6): 622-34.

5 Ali AT. Polycystic ovary syndrome and metabolic syndrome. Ceska Gynekol. 2015 Aug; 80(4):279-89.

6 Kakoly NS, Khomami MB, Joham AE, Cooray SD, Misso ML, Norman RJ, et al. Ethnicity, obesity and the prevalence of impaired glucose tolerance and type 2 diabetes in PCOS: a systematic review and meta-regression. Hum Reprod Update. 2018 Jul 1;24(4):455-67.

7 Szczuko M, Sankowska P, ZapałowskaChwyć M, Wysokiński P. Studies on the quality nutrition in women with polycystic ovary syndrome (PCOS). Rocz Panstw Zakl Hig. 2017;68(1):61-7.

8 Tremellen K, Pearce K. Dysbiosis of gut microbiota (DOGMA): a novel theory for the development of polycystic ovarian syndrome. Med Hypotheses. 2012 Jul;79(1):104-12.

9 Zhou XY, Zhou L, Sun ZY. [Research progress in mechanism of traditional Chinese medicine treatment of polycystic ovary syndrome]. Zhongguo Zhong Yao Za Zhi. 2016 Oct;41(20):3715-20.

10 Wang HCL, Ding Y, Zhu X, Lai J, Jin ZHOU, Li LI, et al. Research progress of tradition Chinese medicine in treatment of polycystic ovary syndrome. Jilin J Chin Med. 2018;38(12): 1485-7.

11 Barrea L, Marzullo P, Muscogiuri G, Di Somma C, Scacchi M, Orio F, et al. Source and amount of carbohydrate in the diet and inflammation in women with polycystic ovary syndrome. Nutr Res Rev. 2018 Dec;31(2): 291-301.

12 Dokras A, Stener-Victorin E, Yildiz BO, Li R Ottey S, Shah D, et al. Androgen excess- polycystic ovary syndrome society: position statement on depression, anxiety, quality of life, and eating disorders in polycystic ovary syndrome. Fertil Steril. 2018 May;109(5):888-99.
$13 \mathrm{Kim} \mathrm{CH}$, Chon SJ, Lee SH. Effects of lifestyle modification in polycystic ovary syndrome compared to metformin only or metformin addition: A systematic review and meta-analysis. Sci Rep. 2020 May 8;10(1):7802.

14 Barrea L, Arnone A, Annunziata G, Muscogiuri G, Laudisio D, Salzano C, et al. Adherence to the mediterranean diet, dietary patterns and body composition in women with polycystic ovary syndrome (PCOS). Nutrients. 2019 Sep 23;11(10).

15 Martinez-Gonzalez MA, Salas-Salvado J, Estruch R, Corella D, Fito M, Ros E, et al. Benefits of the mediterranean diet: insights from the PREDIMED Study. Prog Cardiovasc Dis. 2015 Jul-Aug;58(1):50-60.

16 Ditano-Vazquez P, Torres-Pena JD, GaleanoValle F, Perez-Caballero AI, Demelo-Rodriguez $\mathrm{P}$, Lopez-Miranda J, et al. The fluid aspect of the mediterranean diet in the prevention and management of cardiovascular disease and diabetes: the role of polyphenol content in moderate consumption of wine and olive oil. Nutrients. 2019 Nov 19;11(11): 2833.

17 Mirabelli M, Chiefari E, Arcidiacono B, Corigliano DM, Brunetti FS, Maggisano V, et al. Mediterranean diet nutrients to turn the tide against insulin resistance and related diseases. Nutrients. 2020 Apr 12;12(4):1066.

18 Frias-Toral E, Garcia-Velasquez E, de Los Angeles Carignano M, Rodriguez-Veintimilla D, Alvarado-Aguilera I, Bautista-Litardo N. Polycystic ovary syndrome and obesity: clini$\mathrm{cal}$ aspects and nutritional management. Minerva Endocrinol. 2021 Apr 1. Online ahead of print.

19 Molendijk I, van der Marel S, Maljaars PWJ. Towards a food pharmacy: immunologic modulation through diet. Nutrients. 2019 May 31;11(6):1239.

20 Kazemi M, Jarrett BY, Vanden Brink H, Lin AW, Hoeger KM, Spandorfer SD, et al. Obesity, insulin resistance, and hyperandrogenism mediate the link between poor diet quality and ovarian dysmorphology in reproductive-aged women. Nutrients. 2020 Jun 30; 12(7):1953.

21 Leri M, Scuto M, Ontario ML, Calabrese V, Calabrese EJ, Bucciantini M, et al. Healthy effects of plant polyphenols: molecular mechanisms. Int J Mol Sci. 2020 Feb 13;21(4):1250.
22 Piroddi M, Albini A, Fabiani R, Giovannelli L, Luceri C, Natella F, et al. Nutrigenomics of extra-virgin olive oil: a review. Biofactors. 2017 Jan 2;43(1):17-41.

23 Abenavoli L, Milanović M, Milić N, Luzza F, Giuffrè AM. Olive oil antioxidants and nonalcoholic fatty liver disease. Expert Rev Gastroenterol Hepatol. 2019 Aug;13(8):739-49.

24 Davis C, Bryan J, Hodgson J, Murphy K. Definition of the mediterranean diet; a literature review. Nutrients. 2015 Nov 5;7(11):9139-53.

25 Bach-Faig A, Berry EM, Lairon D, Reguant J, Trichopoulou A, Dernini S, et al. Mediterranean diet pyramid today. Science and cultural updates. Public Health Nutr. 2011 Dec; 14(12A):2274-84.

26 Joshi S, Ostfeld RJ, McMacken M. The ketogenic diet for obesity and diabetes-enthusiasm outpaces evidence. JAMA Intern Med. 2019;179(9):1163-4

27 Puchalska P, Crawford PA. Multi-dimensional roles of ketone bodies in fuel metabolism, signaling, and therapeutics. Cell Metab. 2017 Feb 7;25(2):262-84.

28 Merra G, Miranda R, Barrucco S, Gualtieri P, Mazza M, Moriconi E, et al. Very-low-calorie ketogenic diet with aminoacid supplement versus very low restricted-calorie diet for preserving muscle mass during weight loss: a pilot double-blind study. Eur Rev Med Pharmacol Sci. 2016 Jul;20(12):2613-21.

29 Courchesne-Loyer A, Croteau E, Castellano CA, St-Pierre V, Hennebelle M, Cunnane SC. Inverse relationship between brain glucose and ketone metabolism in adults during short-term moderate dietary ketosis: a dual tracer quantitative positron emission tomography study. J Cereb Blood Flow Metab. 2017 Jul;37(7):2485-93

30 Kuchkuntla AR, Shah M, Velapati S, Gershuni VM, Rajjo T, Nanda S, et al. Ketogenic diet: an endocrinologist perspective. Curr Nutr Rep. 2019 Dec;8(4):402-10.

31 Gupta L, Khandelwal D, Kalra S, Gupta P, Dutta D, Aggarwal S. Ketogenic diet in endocrine disorders: current perspectives. J Postgrad Med. 2017 Oct-Dec;63(4):242-51.

32 Mavropoulos JC, Yancy WS, Hepburn J, Westman EC. The effects of a low-carbohydrate, ketogenic diet on the polycystic ovary syndrome: a pilot study. Nutr Metab. 2005 Dec 16;2:35. 
33 Paoli A, Mancin L, Giacona MC, Bianco A, Caprio M. Effects of a ketogenic diet in overweight women with polycystic ovary syndrome. J Transl Med. 2020 Feb 27;18(1):104.

34 Schugar RC, Crawford PA. Low-carbohydrate ketogenic diets, glucose homeostasis, and nonalcoholic fatty liver disease. Curr Opin Clin Nutr Metab Care. 2012 Jul;15(4): 374-80.

35 Zhang X, Qin J, Zhao Y, Shi J, Lan R, Gan Y, et al. Long-term ketogenic diet contributes to glycemic control but promotes lipid accumulation and hepatic steatosis in type 2 diabetic mice. Nutr Res. 2016 Apr;36(4):349-58.

36 Caprio M, Infante M, Moriconi E, Armani A, Fabbri A, Mantovani G, et al. Very-low-calorie ketogenic diet (VLCKD) in the management of metabolic diseases: systematic review and consensus statement from the Italian society of endocrinology (SIE). J Endocrinol Invest. 2019 Nov;42(11):1365-86.

37 Basciani S, Camajani E, Contini S, Persichetti A, Risi R, Bertoldi L, et al. Very-low-calorie ketogenic diets with whey, vegetable, or animal protein in patients with obesity: a randomized Pilot Study. J Clin Endocrinol Metab. 2020 Sep 1;105(9):dgaa336.

38 Feinman RD, Pogozelski WK, Astrup A, Bernstein RK, Fine EJ, Westman EC, et al. Dietary carbohydrate restriction as the first approach in diabetes management: critical review and evidence base. Nutrition. 2015 Jan; 31(1):1-13.

39 Asemi Z, Esmaillzadeh A. DASH diet, insulin resistance, and serum hs-CRP in polycystic ovary syndrome: a randomized controlled clinical trial. Horm Metab Res. 2015 Mar; 47(3):232-8.

40 Chiu S, Bergeron N, Williams PT, Bray GA, Sutherland B, Krauss RM. Comparison of the DASH (dietary approaches to stop hypertension) diet and a higher-fat DASH diet on blood pressure and lipids and lipoproteins: a randomized controlled trial. Am J Clin Nutr. 2016 Feb;103(2):341-7.

41 Chiavaroli L, Viguiliouk E, Nishi SK, Blanco Mejia S, Rahelić D, Kahleová H, et al. DASH dietary pattern and cardiometabolic outcomes: an umbrella review of systematic reviews and meta-analyses. Nutrients. $2019 \mathrm{Feb}$ $5 ; 11(2): 338$

42 Soltani S, Chitsazi MJ, Salehi-Abargouei A. The effect of dietary approaches to stop hypertension (DASH) on serum inflammatory markers: a systematic review and meta-analysis of randomized trials. Clin Nutr. 2018 Apr; 37(2):542-50.

43 Soltani S, Shirani F, Chitsazi MJ, Salehi-Abargouei A. The effect of dietary approaches to stop hypertension (DASH) diet on weight and body composition in adults: a systematic review and meta-analysis of randomized controlled clinical trials. Obes Rev. 2016 May; 17(5):442-54.
44 Siervo M, Lara J, Chowdhury S, Ashor A, Oggioni C, Mathers JC. Effects of the dietary approach to stop hypertension (DASH) diet on cardiovascular risk factors: a systematic review and meta-analysis. Br J Nutr. 2015 Jan 14;113(1):1-15.

45 Asemi Z, Samimi M, Tabassi Z, Shakeri H, Sabihi SS, Esmaillzadeh A. Effects of DASH diet on lipid profiles and biomarkers of oxidative stress in overweight and obese women with polycystic ovary syndrome: a randomized clinical trial. Nutrition. 2014 Nov-Dec; 30(11-12):1287-93.

46 Foroozanfard F, Rafiei H, Samimi M, Gilasi HR, Gorjizadeh R, Heidar Z, et al. The effects of dietary approaches to stop hypertension diet on weight loss, anti-Mullerian hormone and metabolic profiles in women with polycystic ovary syndrome: a randomized clinical trial. Clin Endocrinol. 2017 Jul;87(1):51-8.

47 Azadi-Yazdi M, Karimi-Zarchi M, SalehiAbargouei A, Fallahzadeh H, Nadjarzadeh A. Effects of dietary approach to stop hypertension diet on androgens, antioxidant status and body composition in overweight and obese women with polycystic ovary syndrome: a randomised controlled trial. J Hum Nutr Diet. 2017 Jun;30(3):275-83.

48 Shang Y, Zhou H, Hu M, Feng H. Effect of diet on insulin resistance in polycystic ovary syndrome. J Clin Endocrinol Metab. 2020 Oct 1; 105(10):dgaa425.

49 Augustin LS, Kendall CW, Jenkins DJ, Willett WC, Astrup A, Barclay AW, et al. Glycemic index, glycemic load and glycemic response: an international scientific consensus summit from the international carbohydrate quality consortium (ICQC). Nutr Metab Cardiovasc Dis. 2015 Sep;25(9):795-815.

50 Barclay AW, Petocz P, McMillan-Price J, Flood VM, Prvan T, Mitchell P, et al. Glycemic index, glycemic load, and chronic disease risk: a meta-analysis of observational studies. Am J Clin Nutr. 2008 Mar;87(3):627-37.

51 Zafar MI, Mills KE, Zheng J, Regmi A, Hu SQ, Gou L, et al. Low-glycemic index diets as an intervention for diabetes: a systematic review and meta-analysis. Am J Clin Nutr. 2019 Oct 1;110(4):891-902.

52 Zhang JY, Jiang YT, Liu YS, Chang Q, Zhao $\mathrm{YH}, \mathrm{Wu}$ QJ. The association between glycemic index, glycemic load, and metabolic syndrome: a systematic review and dose-response meta-analysis of observational studies. Eur J Nutr. 2020 Mar;59(2):451-63.

53 Livesey G, Livesey H. Coronary heart disease and dietary carbohydrate, glycemic index, and glycemic load: dose-response meta-analyses of prospective cohort studies. Mayo Clin Proc Innov Qual Outcomes. 2019 Mar;3(1): 52-69.

54 Graff SK, Mário FM, Alves BC, Spritzer PM. Dietary glycemic index is associated with less favorable anthropometric and metabolic profiles in polycystic ovary syndrome women with different phenotypes. Fertil Steril. 2013 Oct;100(4):1081-8.
55 Szczuko M, Zapalowska-Chwyć M, Drozd R. A low glycemic index decreases inflammation by increasing the concentration of uric acid and the activity of glutathione peroxidase (GPx3) in patients with polycystic ovary syndrome (PCOS). Molecules. 2019 Apr 17; 24(8):1508.

56 Marsh KA, Steinbeck KS, Atkinson FS, Petocz P, Brand-Miller JC. Effect of a low glycemic index compared with a conventional healthy diet on polycystic ovary syndrome. Am J Clin Nutr. 2010 Jul;92(1):83-92.

57 Barr S, Reeves S, Sharp K, Jeanes YM. An isocaloric low glycemic index diet improves insulin sensitivity in women with polycystic ovary syndrome. J Acad Nutr Diet. 2013 Nov; 113(11):1523-31.

58 Sordia-Hernandez LH, Ancer Rodriguez P, Saldivar Rodriguez D, Trejo Guzman S, Servin Zenteno ES, Guerrero Gonzalez G, et al. Effect of a low glycemic diet in patients with polycystic ovary syndrome and anovulation - a randomized controlled trial. Clin Exp Obstet Gynecol. 2016;43(4):555-9.

59 Atiomo W, Read A, Golding M, Silcocks P, Razali N, Sarkar S, et al. Local recruitment experience in a study comparing the effectiveness of a low glycaemic index diet with a low calorie healthy eating approach at achieving weight loss and reducing the risk of endometrial cancer in women with polycystic ovary syndrome (PCOS). Contemp Clin Trials. 2009 Sep;30(5):451-6.

60 Becker GF, Passos EP, Moulin CC. Shortterm effects of a hypocaloric diet with low glycemic index and low glycemic load on body adiposity, metabolic variables, ghrelin, leptin, and pregnancy rate in overweight and obese infertile women: a randomized controlled trial. Am J Clin Nutr. 2015 Dec;102(6):1365-72.

61 Shishehgar F, Mirmiran P, Rahmati M, Tohidi M, Ramezani Tehrani F. Does a restricted energy low glycemic index diet have a different effect on overweight women with or without polycystic ovary syndrome? BMC Endocr Disord. 2019 Sep 2;19(1):93.

62 Douglas CC, Norris LE, Oster RA, Darnell BE, Azziz R, Gower BA. Difference in dietary intake between women with polycystic ovary syndrome and healthy controls. Fertil Steril. 2006 Aug;86(2):411-7.

63 Shishehgar F, Ramezani Tehrani F, Mirmiran P, Hajian S, Baghestani AR, Moslehi N. Comparison of dietary intake between polycystic ovary syndrome women and controls. Glob J Health Sci. 2016 Sep 1;8(9):54801.

64 Matthan NR, Ausman LM, Meng H, Tighiouart $\mathrm{H}$, Lichtenstein $\mathrm{AH}$. Estimating the reliability of glycemic index values and potential sources of methodological and biological variability. Am J Clin Nutr. 2016 Oct;104(4): 1004-13.

65 Mudryj AN, Yu N, Aukema HM. Nutritional and health benefits of pulses. Appl Physiol Nutr Metab. 2014 Nov;39(11):1197-204. 
66 Sievenpiper JL, Kendall CW, Esfahani A, Wong JM, Carleton AJ, Jiang HY, et al. Effect of non-oil-seed pulses on glycaemic control: a systematic review and meta-analysis of randomised controlled experimental trials in people with and without diabetes. Diabetologia. 2009 Aug;52(8):1479-95.

67 Gao R, Duff W, Chizen D, Zello GA, Chilibeck PD. The effect of a low glycemic index pulse-based diet on insulin sensitivity, insulin resistance, bone resorption and cardiovascular risk factors during bed rest. Nutrients. 2019 Aug 27;11(9):2012.

68 Kazemi M, McBreairty LE, Chizen DR, Pierson RA, Chilibeck PD, Zello GA. A comparison of a pulse-based diet and the therapeutic lifestyle changes diet in combination with exercise and health counselling on the cardiometabolic risk profile in women with polycystic ovary syndrome: a randomized controlled trial. Nutrients. 2018 Sep 30;10(10): 1387.

69 Charalampakis V, Tahrani AA, Helmy A, Gupta JK, Singhal R. Polycystic ovary syndrome and endometrial hyperplasia: an overview of the role of bariatric surgery in female fertility. Eur J Obstet Gynecol Reprod Biol. 2016 Dec;207:220-6.

70 Wekker V, van Dammen L, Koning A, Heida KY, Painter RC, Limpens J, et al. Long-term cardiometabolic disease risk in women with PCOS: a systematic review and meta-analysis. Hum Reprod Update. 2020 Nov 1;26(6):94260.
71 Conway G, Dewailly D, Diamanti-Kandarakis E, Escobar-Morreale HF, Franks S, Gambineri A, et al. The polycystic ovary syndrome: a position statement from the European society of endocrinology. Eur J Endocrinol. 2014 Oct;171(4):P1-29.

72 Larsson I, Hulthén L, Landén M, Pålsson E, Janson P, Stener-Victorin E. Dietary intake, resting energy expenditure, and eating behavior in women with and without polycystic ovary syndrome. Clin Nutr. 2016 Feb;35(1):213-8.

73 Brower MA, Hai Y, Jones MR, Guo X, Chen YI, Rotter JI, et al. Bidirectional mendelian randomization to explore the causal relationships between body mass index and polycystic ovary syndrome. Hum Reprod. 2019 Jan 1; 34(1):127-36

74 Tosi F, Bonora E, Moghetti P. Insulin resistance in a large cohort of women with polycystic ovary syndrome: a comparison between euglycaemic-hyperinsulinaemic clamp and surrogate indexes. Hum Reprod. 2017 Dec 1; 32(12):2515-21.

75 Escobar-Morreale HF. Polycystic ovary syndrome: definition, aetiology, diagnosis and treatment. Nat Rev Endocrinol. 2018 May; 14(5):270-84

76 Muscogiuri G, Barrea L, Caprio M, Ceriani F, Chavez AO, El Ghoch M, et al. Nutritional guidelines for the management of insulin resistance. Crit Rev Food Sci Nutr. 2021 Apr 2: 1-14. Epub ahead of print.

77 Porchia LM, Hernandez-Garcia SC, Gonzalez-Mejia ME, López-Bayghen E. Diets with lower carbohydrate concentrations improve insulin sensitivity in women with polycystic ovary syndrome: a meta-analysis. Eur J Obstet Gynecol Reprod Biol. 2020 May;248:110-7.

78 Greenland P. Dietary adherence in a clinical trial of a nutritional and behavioral intervention. JAMA. 2019 Oct 15;322(15):1500.

79 Moran LJ, Ranasinha S, Zoungas S, McNaughton SA, Brown WJ, Teede HJ. The contribution of diet, physical activity and sedentary behaviour to body mass index in women with and without polycystic ovary syndrome. Hum Reprod. 2013 Aug;28(8):2276-83.

80 Moran LJ, Grieger JA, Mishra GD, Teede HJ. The association of a mediterranean-style diet pattern with polycystic ovary syndrome status in a Community Cohort Study. Nutrients. 2015 Oct 16;7(10):8553-64.

81 Sayer RD, Peters JC, Pan Z, Wyatt HR, Hill JO. Hunger, food cravings, and diet satisfaction are related to changes in body weight during a 6-month behavioral weight loss tntervention: the beef WISE Study. Nutrients. 2018 May 31;10(6):700.

82 Gibson AA, Seimon RV, Lee CM, Ayre J, Franklin J, Markovic TP, et al. Do ketogenic diets really suppress appetite? A systematic review and meta-analysis. Obes Rev. 2015 Jan; 16(1):64-76.

83 Muscogiuri G, Barrea L, Laudisio D, Pugliese G, Salzano C, Savastano S, et al. The management of very low-calorie ketogenic diet in obesity outpatient clinic: a practical guide. J Transl Med. 2019 Oct 29;17(1):356. 